\title{
Target Detection System Design based on Mini2440 Platform
}

\author{
Wei Xue ${ }^{a}$, Yiheng Zhang ${ }^{b}$ \\ College of Information and Communication Engineering, Harbin Engineering University, Harbin, \\ China

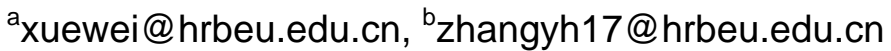

Keywords: mini2440, background difference method, normalized mathematical method, graying arithmetic, target detection.

\begin{abstract}
According to the target detection of the portable platform, we put forward a design of system. The algorithm is based on background difference method which combines normalized mathematical method, graying arithmetic and morphological method to deal with. Experiments show that we can achieve static or moving target detection in a static background. The method proposed in this paper has robustness. And it has simple principle and low calculation. It is perfect for the portable platform.
\end{abstract}

\section{Introduction}

Target detection has extensive application in the normal life and actual production. We cannot leave the target detection in safety monitoring, supervision of the production, the traffic control and military. The existing target detection systems mostly have complex arithmetic and great computational complexity. They are not suitable for use on a smaller scale embedded platforms. In this paper, we used the mini2440 development board produced by the company which named Friendly ARM and CAM130 camera module as hardware systems. Then we designed a simple and convenient method on this basis to satisfy the requirements of target detection, and used $\mu \mathrm{C} / \mathrm{OS}-\mathrm{II}$ real-time operating system to schedule tasks to achieve the goal of multi-functional target detection.

\section{Mini2440 Development Board Introduction}

Mini2440 development board is a low-cost and practical ARM9 development board which produced by the company which named Friendly ARM. It uses a Samsung S3C2440 chip as the microprocessor, and has a high cost and stability.

S3C2440 microprocessor has many functions module. In this system, we primarily use the LCD controller and camera interface.

\section{Target Detection Algorithms}

There are three common target detection algorithms. These algorithms respectively are optical flow method, inter-frame difference method and background difference method.

Moving target detection based on optical flow method uses the feature that moving targets show as velocity field in the image when they change with time, then calculates the optical flow corresponding to the current motion according to certain constraints.

The basic idea of the inter-frame difference method is to compare the inter-frame difference between adjacent two frames, and to detect the target with the relatively strong correlation between two adjacent frames. The whole operation process can be shown in flow chart Fig. 1.

The basic idea of background difference method is to subtract the background images from the current frame. Then use the relationship between the current image and the background image to detect the target. The whole operation process can be shown in flow chart Fig. 2. 


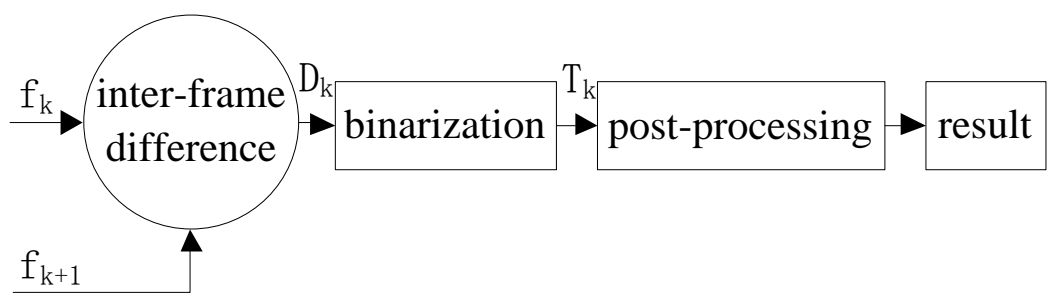

Fig.1 Inter-frame difference method process

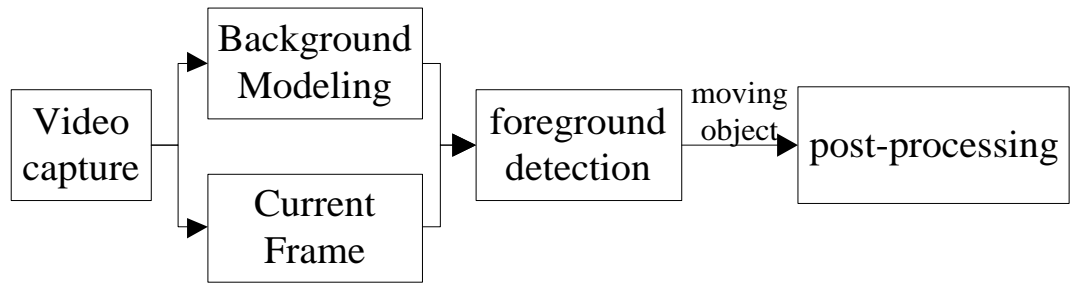

Fig.2 Background difference method process

Of these three methods, the optical flow method requires multiple operations. There are little people uses this method in real-time system. On the contrary, the inter-frame difference method and the background subtraction are used more for its speed calculating and accurate detection. Therefore, we use the background difference method in this system as a target detection algorithm.

\section{Preprocessing and Post-processing Algorithms}

When we detect the target, there will be a lot of noise interference in the test results. In order to improve the quality of the detection, it is necessary to reduce the effect of these noises. Therefore, it must be some simple and reliable pre-processing and post-processing.

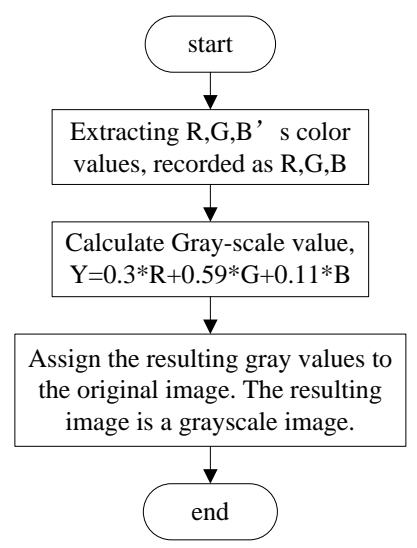

Fig.3 Gray processing

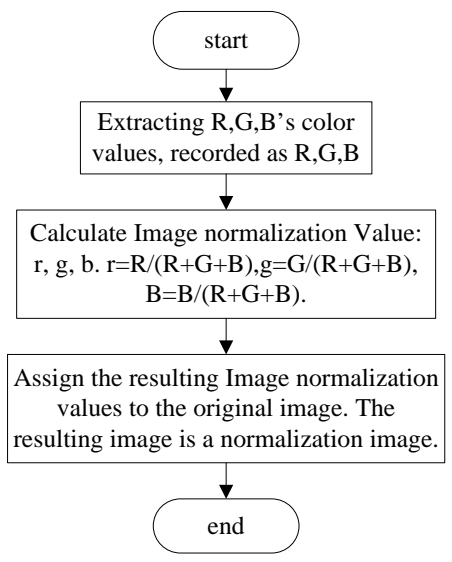

Fig.4 Image normalization

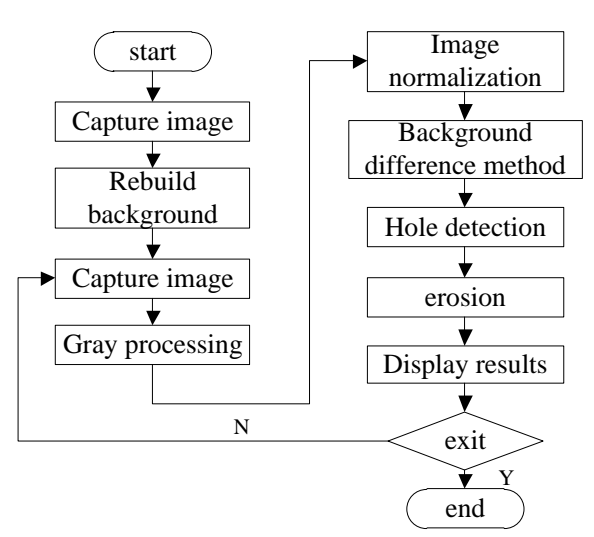

Fig.5 Operation process

\section{Color Image Gray Processing}

The images what we collected with the camera is in color. It is difficult to set the threshold when we directly process color images. This will make detection have more noise, and even will appear error detection or undetected. However the gray-scale image only has 256 gray levels. Its color change will be much less than the color image. Meanwhile, the gray-scale images and color images both can reflect image's whole and partial chrominance and luminance information. So using grayscale image to process will be a great advantage during the background difference. The whole operation process of color image gray processing can be shown in flow chart Fig. 3. 


\section{Whole Detection}

Because of the uncertainty noise in the image, there will be many black pixel spots in the white portion when we detect moving objects. These black pixel spots are the holes. In order to improve the detection effect, we must be detected and filled these holes. So we need to use Morphological Operation of whole detection. The basic idea of whole detection is as follows:

1. Detect the black pixel;

2. Scan the pixel which be processed with the structuring elements;

3. Calculate the binary image and the structuring elements;

4. Record the value of that calculation result is 1 . Set a threshold value. When the calculation result is bigger than the threshold, the point is the holes which need to be filled, and the value need to be set to 1 . Otherwise, the point is the background portion, and we don't need to do anything.

5. Traverse black pixels of the whole image. The result is the result of the hole detected.

\section{Erosion}

There will be salt noise in the detection image after the whole detection. At the same time, the contour of the detection target also will have some glitches. In order to extract the key information of the image and remove the interference, we can use erosion.

If $\mathrm{Z}$ is a collection of $\mathrm{A}, \mathrm{B}$, the operation steps of erosion using $\mathrm{B}$ to $\mathrm{A}$ is as follows:

1. Scan every pixel of A, the image to be processed with structural element B.

2. Perform and operation with the structural elements and the binary image which it covered.

3. If the calculation result is 1 , this pixel value of the result image is 1 , otherwise is 0 . In practical applications, in order to remove the effects of certain noise, we are generally not finds that the pixel is 1 when the results all are 1 , but set a threshold. When the result of the calculation is greater than this threshold we will determine that the pixel 1 , otherwise we will determine 0 .

\section{System Design and Implementation}

The system is divided into four parts module: image acquisition, pre-processing module, object detection module and post-processing module.

Image acquisition module is used to extract image information. When the camera sends the captured images to ARM, ARM will receive them by the camera interface module.

Preprocessing module is mainly used to reduce the impact of the diversity of color and light caused. This module includes color image gray processing and image normalization two parts. While in the first pre-processing, we need to rebuild the background.

In the target detection module, we use background difference method to process the image.

Post-processing is used to reduce the salt-pepper noise of the processed image, and remove interference, improve the detection results. This module includes whole detection and erosion.

The whole operation process can be shown in flow chart Fig. 5.

\section{Experimental Results and Analysis}

The system is written in C programs. Hardware platform uses the mini2440 ARM development board produced by Friendly ARM Company. Video images uses RGB565 format. Image size is $320 * 240$ (pixels). The experimental results are shown in flow chart Fig.6.

\section{Conclusion}

This article relates to the moving target detection algorithm that make the background difference method as the core of and the related pre-processing and post-processing algorithm. And introduce the principle of these algorithms and the implementation. Due to the limited computing power of the 
chip, the system needs to continue to optimize algorithm, and improve the real-time and robustness of the system. This is the future direction of improvement of the system.

(a) The first image (b) the first result(c) the second image (d) the second result (e) the third image

(f)the third result(g)the fourth image(h)the fourth result(i)the fifth image(j)the fifth result

(k) The sixth image (l) the sixth result (m) the seventh frame image (n) the seventh result (o) background

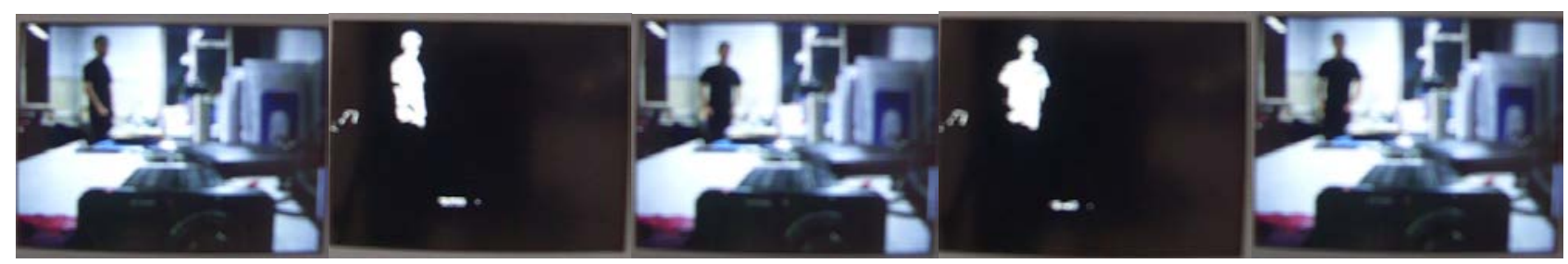

(a)

(b)

(c)

(d)

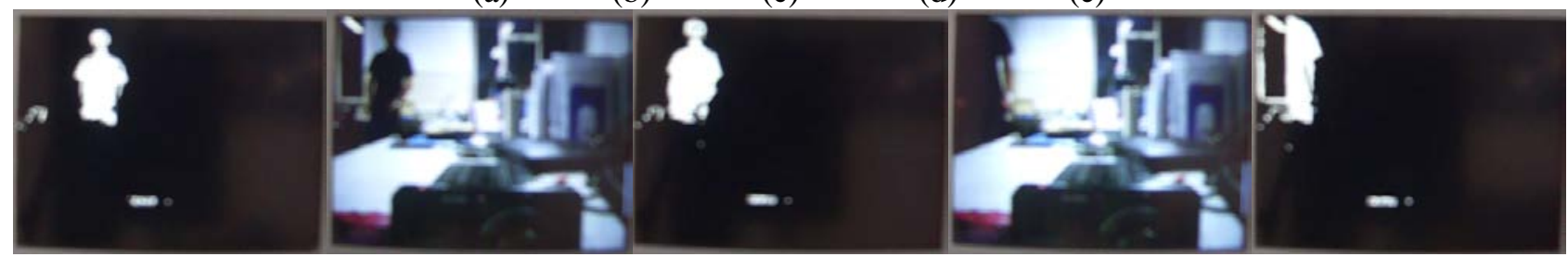

(f)

(g)

(h)

(i)

(j)

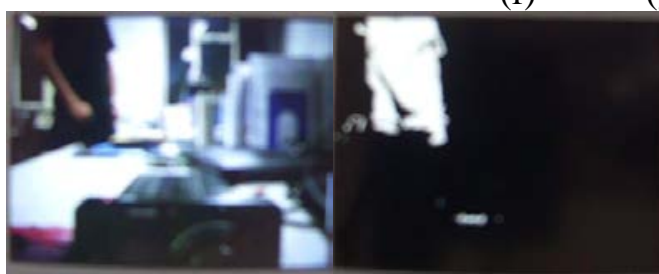

(k)

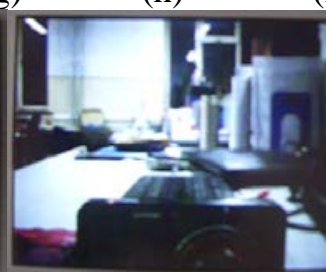

(l) (m)

Fig.6 Detection result

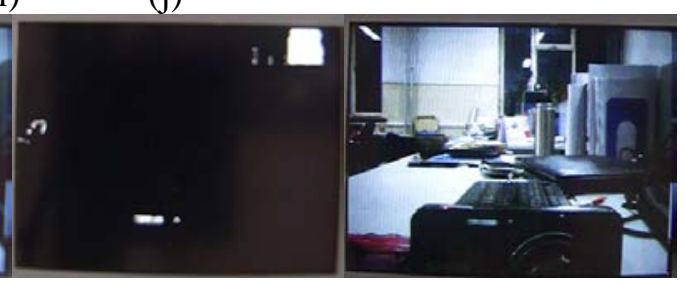

(n) (o)

\section{Acknowledgments}

This paper is funded by the International Exchange Program of Harbin Engineering University for Innovation-oriented Talents Cultivation, International Science \& Technology Cooperation Program of China (2014 DFR10240), National Natural Science Foundation of China (612111070), China Postdoctoral Science Foundation (2013T60346), Harbin science and technology research projects (2013AE1BE003), Heilongjiang province natural science foundation projects (F201344).

\section{References}

[1] Yu Linan, Moving target detection and tracking system based on a single camera and ARM platform, Ph.M. Dissertation, Dalian, 2010.

[2] Qiao Shikun, Stationary background moving target detection technology based on boundary, Ph.M. Dissertation, Harbin, 2007.

[3] Han Xiaowei, Key Technology Research on color image processing, Ph.M. Dissertation, Shenyang, 2005.

[4] S3C2440A 32 bit CMOS RISC Micro controller user manual.

[5] Tian Bing, Li Qin, Sun Meng: submitted to Customer of Instrument and Meter (2007) 